\title{
Erratum to: Positive alphas and a generalized multiple-factor asset pricing model
}

\author{
Robert Jarrow ${ }^{1,2}$. Philip Protter ${ }^{3}$
}

Published online: 3 November 2015

(C) Springer-Verlag Berlin Heidelberg 2015

\section{Erratum to: Math Finan Econ DOI 10.1007/s11579-015-0149-1}

In our published paper "Positive alphas and a generalized multiple-factor asset pricing model" we regret that there was a mistake. In Theorem 6 of that paper we claim a condition is necessary and sufficient. In fact it is only sufficient for there to exist an arbitrage opportunity, in the sense that there does not exist an equivalent local martingale measure. This is the more interesting implication. We offer the following as a replacement for Theorem 6 in the original text.

Theorem 6 (Non-zero Alphas) A non-zero alpha $\alpha_{v}(t) \neq 0$ implies there does not exist an equivalent martingale measure for the primary traded assets.

By a non-zero alpha what we mean in the technical sense is that $\alpha_{v}(t)$ is an optional process that is distinguishable from the zero process. This means that there exists a (possibly and usually non unique) stopping time $\tau$ such that $\left|\alpha_{v}\left(\tau 1_{\{\tau<\infty\}}\right) 1_{\{\tau<\infty\}} \neq 0\right|$ with positive probability.

Proof Theorem 5 proves that the existence of an equivalent martingale measure implies alpha is zero. Hence, a non-zero alpha implies there does not exist an equivalent martingale measure. This completes the proof.

The online version of the original article can be found under doi:10.1007/s11579-015-0149-1.

Robert Jarrow

raj15@cornell.edu

1 Samuel Curtis Johnson Graduate School of Management, Cornell University, Ithaca, NY 14853, USA

2 Kamakura Corporation, Honolulu, USA

3 Statistics Department, Columbia University, New York, NY 10027, USA 